Abstract

\title{
Detection of Internal Defects in Concrete and Evaluation of a Healthy Part of Concrete by Non- Contact Acoustic Inspection Using Normalized Spectral Entropy ${ }^{\dagger}$
}

\author{
Kazuko Sugimoto and Tsuneyoshi Sugimoto \\ Graduate School of Engineering, Toin University of Yokohama, Yokohama, Japan \\ † Presented at the Entropy 2021: The Scientific Tool of the 21st Century, 5-7 May 2021; Available online: \\ https://sciforum.net/conference/Entropy2021/.
}

Published: 5 May 2021

In recent years, deterioration of concrete structures has become a social problem, and there is a demand for a non-contact, non-destructive method for inspecting internal defects in concrete structures. In our noncontact acoustic inspection, a target surface of concrete is vibrated with strong aerial sound waves, the vibration velocity distribution is measured two-dimensionally using a scanning laser Doppler vibrometer. Then, after time-frequency gate process, acoustic features quantities (vibrational energy ratio and spectral entropy) are calculated. By analysis using them, it has become possible to detect and visualize internal defects from a long distance $(5-30 \mathrm{~m})$. Traditional spectral entropy has no problem in evaluating the fluctuation of spectral entropy value within the same measurement condition or a measurement plane. However, it was not possible to directly compare and evaluate the spectral entropy values because the meaning of the upper and lower limit of spectral entropy values are ambiguous between different measurement conditions and different objects. To solve this, we introduce normalized spectral entropy. It will become possible to compare the values in different measurement conditions and different objects on the same scale, and it becomes possible to statistically evaluate a healthy part of concrete as well as detect defects.

(C) 2021 by the authors. Licensee MDPI, Basel, Switzerland. This article is an open access article distributed under the terms and conditions of the Creative Commons Attribution (CC BY) license (http://creativecommons.org/licenses/by/4.0/). 